\title{
Vectorial Integrated Finite-Difference Analysis of Dielectric Waveguides
}

\author{
Haozhe Dong, Member, IEEE, Anthony Chronopoulos, Junping Zou, Member, IEEE, and Anand Gopinath
}

\begin{abstract}
An integrated finite difference approach is formulated for the full vector solution using transverse magnetic field components for dielectric waveguides, which is particularly suitable for nonuniform mesh and internal flux boundary conditions. This approach creates a sparse banded asymmetric matrix. Only few largest positive eigenvalues and the corresponding eigenvectors are calculated by the Arnoldi method (based on the modified Gram-Schmidt) coupled with multiple deflation by computing a suitable small size matrix. The Arnoldi process is followed by an inverse power method combined with an iterative solver. The nonphysical modes have been excluded by applying the divergence relation $\nabla \cdot H=0$. Three numerical examples are calculated for verifying the reliability and efficiency of this technique, the first two of them are used for the comparison with the results obtained by other methods, and last one is a quantum well single mode optical waveguide. The technique in this paper could be used for any shape of dielectric waveguides with any profile of refractive index in the cross section plane with proper Taylor expansion of the index.
\end{abstract}

\section{INTRODUCTION}

D IELECTRIC waveguides are of interest in a variety of integrated microwave circuits and optical circuits. The guided modes are the very basic physical variable to be known. Analysis of the modes of dielectric waveguides is necessary for various design. In most cases, the analytical solution for the modes is not available, thus the use of numerical analysis becomes essential. For the step index profile of dielectric waveguides, the mode-matching techniques [1], the finiteelement analysis [2]-[4], and the finite-difference method [5]-[7] have been developed for the discretization of the wave equation and the computation of the eigenvalues. In this paper, we present an integrated finite-difference method [8] to discretize the wave equation, and the Arnoldi method [9], [10] coupled with multiple deflation [10], followed by the inverse power method [11] combined with an iterative solver [12], for the calculation of eigenvalues and eigenvectors.

The integrated finite-difference approach [8] is formulated in terms of transverse components of the vector magnetic $(H)$ field. A nonuniform mesh is used in the cross section of waveguides in this approach. The wave equation for each component

Manuscript received December 4, 1992; revised March 25, 1993. This work was supported in part by DARPA Contract under AF/F19628-91-K-0044. The Minnesota Supercomputing Institute (MSI) provided the Cray computing time. H. Dong and A. Gopinath are with the Department of Electrical Engineering, University of Minnesota, Minneapolis, MN 55455.

A. Chronopoulos is with the Department of Computer Sciences, University of Minnesota, Minneapolis, MN 55455.

J. Zou is with Cray Research Incorporation, Chippewa Falls, WI 54729.

IEEE Log Number 9210189. of the $H$ field is integrated over each cell of the mesh by the box integration method [8] in the finite difference scheme to discretize the wave equation. The boundary condition at the interface with the nearest neighbor cells is enforced by employing the transverse and longitudinal continuity of the $H$ field. The discretization of these boundary conditions together with the wave equation creates a sparse banded asymmetric matrix, which has two diagonal sections for $H_{x}$ and $H_{y}$, and two off-diagonal sections for the coupling between the $H_{x}$ and $H_{y}$. The exponential decay of the $H$ field at the clad layers is accounted for by choosing a nonuniform mesh at those regions, but the mesh of the guide region is uniform. Only the nonzero elements of the matrix are stored.

The size of the matrix with coupling between the transverse components is about four times larger than the matrix without this coupling. Solving for all the eigenvalues of the matrix for a large number of nodes is too computer intensive. Since only few positive eigenvalues need to be obtained, depending on the mode structure of the waveguide, finding a method for accurately computing few eigenvalues of the matrix is highly desirable. We use the modified Arnoldi method [9] with multiple deflation to calculate few extreme eigenvalues and the corresponding eigenvectors by computing a suitable small size matrix. With the multiple deflation [10], the calculation of eigenvalues only follows the few desired ones. For the strongly guided modes, the convergence of the calculation is fast and accurate. But for single mode waveguides or weakguided modes, improvement of convergence of the calculation is needed for rapid convergence. We use the inverse power method [11] to continue the calculation after the Arnoldi iteration gives us the initial eigenvalues. This strategy makes the convergence of the calculation very fast. The $s$-step method [12] is combined with the inverse power method to avoid directly inverting the original matrix.

We consider three examples. The first two dielectric waveguides discussed in the literature have strong guided modes. The last example is for one of a single mode three quantum well optical waveguide structure.

The Section II shows the discretization process, and the Section III gives the description of numerical technique and the calculation strategy. The examples are shown in Section IV, and the conclusion is in Section V.

\section{DisCRETIZATION}

Wave propagation in an inhomogeneous dielectric waveguide may be formulated in terms of longitudinal components of $E$ or $H$ fields, or alternatively using transverse components 


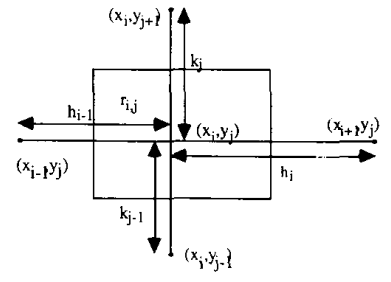

Fig. 1. A cell of box integration method.

[13]. In order to eliminate all spurious modes, the zero divergence relation of $H$ field must be enforced in the solving of wave equation. The formulation in terms of the transverse components $H_{x}$ and $H_{y}$ of $H$ field may be used to advantage [13] to circumvent the spurious modes.

We assume a harmonic wave propagation, $\exp (-j \beta z)$ in the axial direction ( $z$-direction), where the $\beta$ is the propagation constant. The $z$-component dependent can be separated from the $x$ and $y$ components. For each region of constant index of refraction, the Helmholtz wave equation becomes:

$$
\begin{aligned}
& \nabla^{2} H_{x}+(k n(x, y))^{2} H_{x}=\beta^{2} H_{x} \\
& \nabla^{2} H_{y}+(k n(x, y))^{2} H_{y}=\beta^{2} H_{y}
\end{aligned}
$$

where $H_{x}$ and $H_{y}$ are the transverse components of the $H$ field, $k$ is the wave number in free space, $n(x, y)$ is the index of refraction, and $\nabla=(\partial / \partial x, \partial / \partial y)$. We divide the cross section into rectangular cells, and each cell is divided into four regions based on a box integration method [8] (see Fig. 1). Each region is assumed to be a constant index of refraction. We integrate (1) and (2) over each region as following:

$$
\int_{c_{i, j}} H_{a} \cdot n d s+\iint_{r_{i, j}}\left(k^{2} n^{2}-\beta^{2}\right) H_{a} d x d y=0
$$

where $a$ is $x$ and $y, c_{i, j}$ is the boundary of region $r_{i, j}, n$ is the unit outward normal of $c_{i, j}$.

The transverse and longitudinal continuity of the $H$ field and the longitudinal continuity of the electric field are used to meet the interface continuity boundary conditions at each internal boundary in a cell as shown in Fig. 1. The longitudinal components of the $H$ field and electrical $(E)$ field can be expressed in terms of $H_{x}$ and $H_{y}$ by using

$$
\nabla \cdot H=\partial H_{x} / \partial x+\partial H_{y} / \partial y-i \beta H_{z}=0
$$

and

$$
\partial H_{y} / \partial x-\partial H_{x} / \partial y=i \omega \epsilon E_{z}
$$

where $\epsilon$ is the dielectric constant, and $\omega$ is angular frequency. The longitudinal continuity of the $H$ and the $E$ along the each interface between region $i$ and $j$ can be expressed as

$$
\begin{aligned}
\frac{1}{\epsilon_{i}}\left(\partial H_{y} / \partial x-\partial H_{x} / \partial y\right) & =\frac{1}{\epsilon_{j}}\left(\partial H_{y} / \partial x-\partial H_{x} / \partial y\right) \\
\left(\partial H_{x} / \partial x+\partial H_{y} / \partial y\right) & =\left(\partial H_{x} / \partial x+\partial H_{y} / \partial y\right)
\end{aligned}
$$

at each interface.
Combining the (3), (6), and (7) by using finite-difference formula as indicated in an box-integration method [8], we discretize the wave equations for the $H_{x}$ and $H_{y}$ as following:

$$
\begin{aligned}
& \frac{1}{2 k_{j}}\left(h_{i-1} \frac{n_{2}}{n_{1}}+h_{i} \frac{n_{3}}{n_{4}}\right) H_{x}(i, j+1) \\
& +\frac{1}{2 k_{j-1}}\left(h_{i-1} \frac{n_{2}}{n_{1}}+h_{i} \frac{n_{3}}{n_{4}}\right) H_{x}(i, j-1) \\
& +\frac{1}{4 h_{i-1}}\left[k_{j}\left(\frac{n_{2}}{n_{1}}+\frac{n_{3}}{n_{4}}\right)\right. \\
& \left.+k_{j-1}\left(\frac{n_{2}}{n_{1}}+\frac{n_{3}}{n_{4}}\right)\right] H_{x}(i-1, j) \\
& +\frac{1}{4 h_{i}}\left[k_{i}\left(\frac{n_{2}}{n_{1}}+\frac{n_{3}}{n_{4}}\right)\right. \\
& \left.+k_{j-1}\left(\frac{n_{1}}{n_{2}}+\frac{n_{4}}{n_{3}}\right)\right] H_{x}(i+1, j) \\
& -\left[\frac{1}{2 k_{j}}\left(h_{i-1} \frac{n_{2}}{n_{1}}+h_{i} \frac{n_{3}}{n_{4}}\right)\right. \\
& +\frac{1}{2 k_{j-1}}\left(h_{i-1} \frac{n_{2}}{n_{1}}+h_{i} \frac{n_{3}}{n_{4}}\right) \\
& +\frac{k_{j}}{4 h_{i-1}}\left(\frac{n_{2}}{n_{1}}+\frac{n_{3}}{n_{4}}\right)+\frac{k_{j-1}}{4 h_{i-1}}\left(\frac{n_{2}}{n_{1}}+\frac{n_{3}}{n_{4}}\right) \\
& +\frac{k_{j}}{4 h_{i}}\left(\frac{n_{2}}{n_{1}}+\frac{n_{3}}{n_{4}}\right)+\frac{k_{j-1}}{4 h_{i}}\left(\frac{n_{1}}{n_{2}}+\frac{n_{4}}{n_{3}}\right) \\
& \left.+\frac{k^{2}}{4}\left(k_{j-1}+k_{j}\right)\left(h_{i-1} n_{1} n_{2}+h_{i} n_{3} n_{4}\right)\right] H_{x}(i, j) \\
& +0.5\left(\frac{n_{2}}{n_{1}}-\frac{n_{1}}{n_{2}}\right) H_{y}(i-1, j) \\
& +0.5\left(\frac{n_{4}}{n_{3}}-\frac{n_{3}}{n_{4}}\right) H_{y}(i+1, j) \\
& -\left[0.5\left(\frac{n_{2}}{n_{1}}-\frac{n_{1}}{n_{2}}\right)+0.5\left(\frac{n_{4}}{n_{3}}-\frac{n_{3}}{n_{4}}\right)\right] H_{y}(i, j) \\
& =\beta^{2}\left[\frac{h_{i-1}}{4}\left(k_{j} \frac{n_{2}}{n_{1}}+k_{j-1} \frac{n_{1}}{n_{2}}\right)\right. \\
& \left.+\frac{h_{i}}{4}\left(k_{j} \frac{n_{3}}{n_{4}}+k_{j-1} \frac{n_{4}}{n_{3}}\right)\right] H_{x}(i, j)
\end{aligned}
$$

$$
\begin{aligned}
\frac{1}{2 h_{i-1}}( & \left.k_{i-1} \frac{n_{3}}{n_{2}}+k_{j} \frac{n_{4}}{n_{1}}\right) H_{y}(i-1, j) \\
& +\frac{1}{2 h_{i}}\left(k_{i-1} \frac{n_{2}}{n_{3}}+h_{i} \frac{n_{1}}{n_{4}}\right) H_{y}(i+1, j) \\
& +\frac{1}{4 k_{j-1}}\left[h_{i-1}\left(\frac{n_{3}}{n_{2}}+\frac{n_{4}}{n_{1}}\right)+h_{i}\left(\frac{n_{2}}{n_{3}}+\frac{n_{1}}{n_{4}}\right)\right] \\
& +H_{y}(i, j-1) \\
& +\frac{1}{4 k_{j}}\left[h_{i-1}\left(\frac{n_{3}}{n_{2}}+\frac{n_{4}}{n_{1}}\right)\right. \\
& \left.+h_{i}\left(\frac{n_{2}}{n_{3}}+\frac{n_{1}}{n_{4}}\right)\right] H_{y}(i, j+1) \\
& -\left[\frac{1}{2 h_{i-1}}\left(k_{i-1} \frac{n_{3}}{n_{2}}+k_{j} \frac{n_{4}}{n_{1}}\right)\right. \\
& +\frac{1}{2 h_{i}}\left(k_{i-1} \frac{n_{2}}{n_{3}}+h_{i} \frac{n_{1}}{n_{4}}\right)
\end{aligned}
$$




$$
\begin{aligned}
& +\frac{h_{i}}{4 k_{j-1}}\left(\frac{n_{3}}{n_{2}}+\frac{n_{4}}{n_{1}}\right)+\frac{h_{i}}{4 k_{j-1}}\left(\frac{n_{2}}{n_{3}}+\frac{n_{1}}{n_{4}}\right) \\
& +\frac{h_{i}}{4 k_{j-1}}\left(\frac{n_{3}}{n_{2}}+\frac{n_{4}}{n_{1}}\right)+\frac{h_{i}}{4 k_{j-1}}\left(\frac{n_{2}}{n_{3}}+\frac{n_{1}}{n_{4}}\right) \\
& \left.+\frac{k^{2}}{4}\left(h_{i-1}+h_{i}\right)\left(k_{j-1} n_{3} n_{2}+k_{j} n_{1} n_{4}\right)\right] H_{y}(i, j) \\
& -0.5\left(\frac{n_{3}}{n_{2}}-\frac{n_{2}}{n_{3}}\right) H_{x}(i, j-1)-0.5\left(\frac{n_{1}}{n_{4}}-\frac{n_{4}}{n_{1}}\right) \\
& +p d H_{x}(i, j+1) \\
& +\left[0.5\left(\frac{n_{3}}{n_{2}}-\frac{n_{2}}{n_{3}}\right)+0.5\left(\frac{n_{1}}{n_{4}}-\frac{n_{4}}{n_{1}}\right)\right] \\
& =H_{x}(i, j) \\
& =\beta^{2}\left[\frac{k_{j-1}}{4}\left(h_{i-1} \frac{n_{3}}{n_{2}}+h_{i} \frac{n_{2}}{n_{3}}\right)\right. \\
& \left.+\frac{k_{j}}{4}\left(h_{i-1} \frac{n_{4}}{n_{1}}+h_{i} \frac{n_{1}}{n_{4}}\right)\right] H_{y}(i, j) .
\end{aligned}
$$

We restate the (8) and (9) in term of matrix as

$$
\boldsymbol{C} U=\lambda \boldsymbol{B} U
$$

or

$$
\left[\begin{array}{ll}
C_{x x} & C_{y x} \\
C_{x y} & C_{y y}
\end{array}\right]\left[\begin{array}{l}
H_{x} \\
H_{y}
\end{array}\right]=\lambda\left[\begin{array}{ll}
B_{x x} & \\
& B_{y y}
\end{array}\right]\left[\begin{array}{l}
H_{x} \\
H_{y}
\end{array}\right]
$$

where $C_{x x}$ and $C_{y y}$ are a five diagonal matrix, $C_{x y}$ and $C_{y x}$ are a three-diagonal matrix, and both of $B_{x x}$ and $B_{y y}$ are a one-diagonal matrix. This eigenvalue problem can be easily transferred to standard eigenvalue problem as

$$
\boldsymbol{A} x=\lambda x
$$

where $\boldsymbol{A}=\boldsymbol{B}^{-1} \boldsymbol{C}$ and $x=U$.

\section{NUMERICAL TECHNIQUE}

For a dielectric waveguide, only the guided modes are of significance, which implies that few largest positive eigenvalues are desired. The Arnoldi method [9] can be used to compute efficiently few eigenvalues and the corresponding eigenvectors of the matrix $\boldsymbol{A}$ of size $n \times n$ for the guided modes.

The Arnoldi algorithm is based on the Arnoldi recursion for reducing a real asymmetric matrix $\boldsymbol{A}$ to upper Hessenberg matrix. The basic Arnoldi procedure can be viewed as the Gram-Schmidt orthogonalization of the Krylov subspace basis $\left\{q_{1}, A q_{1}, \cdots, A_{m-1} q_{1}\right\}$. Furthermore, for each $m$

$$
H_{m}=Q_{m}^{T} A Q_{m}
$$

is the orthogonal projection of $A$ onto the subspace spanned by the Arnoldi vectors $Q_{j}=\left\{q_{1}, \cdots, q_{m}\right\}$ such that $Q_{m} T Q_{m}=$ $I_{m}$ where $I_{m}$ is the identity matrix of order $m$. The upper Hessenberg $H_{m}$ is formed in the Arnoldi algorithm. The eigenvalues of the upper Hessenberg matrices $H_{m}$ are called Ritz values of $A$ (in $Q_{m}$ ). Several of the extreme eigenvalues of $A$-that is, several of the algebraically largest or algebraically smallest of the eigenvalues of $A$-are well approximated by eigenvalues of the matrices $H_{m}$. The Ritz vector $Q_{m} Z(=$ $x$ ) obtained from an eigenvector $Z$ of a given $H_{m}$ is an approximation to a corresponding eigenvector of $A$. Based on the Arnoldi method [9], we form matrices $\boldsymbol{H}$ of size $m \times m$ and matrix $\boldsymbol{V}$ of size $n \times m$, where $m$ is much smaller than $n$. The extreme eigenvalues $\lambda_{1}^{m}$ of $\boldsymbol{H}$ are approximations of the extreme eigenvalues of $\boldsymbol{A}$. Let $\mu$ be an extreme eigenvalue (of largest or smallest size) of $\boldsymbol{H}$ and $Z$ the corresponding eigenvector. The $\mu$ is an approximation to an extreme eigenvalue of $\boldsymbol{A}$ and $\boldsymbol{V Z}$ is its corresponding eigenvector. The error predicted from the Arnoldi method [14] can be evaluated by

$$
\left\|(A-\mu I) V Z_{i}\right\|=h_{m+1, m}\left|e_{m}^{T} Z_{i}\right| \quad i=1, \cdots, m
$$

where $e_{m}^{T}=(0, \cdots, 1)$ and $Z_{i}$ is the ith eigenvector of $\boldsymbol{H}$. This evaluation can be used as a stopping criterion.

After having obtained the first largest positive eigenvalue $\lambda_{1}$, we could compute the second largest eigenvalue by using a deflation [10] process. Let

$$
A_{1}=A-\lambda_{1}^{m}\left(V Z_{1}\right)^{T} V Z_{1} .
$$

As a consequence of the Schur-Wieland [10] deflation the largest of $A_{1}$ is an approximation to the second largest positive eigenvalue of $A$. Repeating this process we can approximate more accurately several positive eigenvalues of the matrix $\boldsymbol{A}$.

For a diagonally dominant matrix (or strong guided modes), the convergence of the Arnoldi process is rapid. But for the case where diagonal dominance no longer exists (i.e., weak guided modes), the acceleration of the convergence of the eigenvalue calculation is needed. We accelerate the modified Amoldi process by the inverse power method [11]. We first apply the Arnoldi method to get an approximation to the (current) largest positive eigenvalue $\mu$ and the corresponding eigenvector $Z$. We then apply the inverse power method by iteratively solving linear systems of the form

$$
(A-\mu I) x=b
$$

using the $s$-step orthomin $(k)$ method [12]. The standard Orthomin $(k)$ did not work well due to the lack of diagonal dominance (in $\boldsymbol{A}$ ). This inverse power technique enhances significantly the accuracy in the approximation of the eigenvalues and eigenvectors.

\section{RESULTS}

In this paper, we present numerical results for three different dielectric waveguide structures. The first two are representative dielectric waveguides and selected for the comparison with previously published numerical approaches [5]-[7]. The spacing of the mesh in the clad layers is exponentially increased, and uniform in the guided region. We use $64 \times 64$ nodes for all of the three structures. All the computations are carried out on the CRAY X-MP.

The first example is a square dielectric waveguide (see Fig. 2). The transverse structure is invariant under $90^{\circ}$ rotation, so the first two largest positive eigenvalues, $H_{11}^{x}$ and $H_{11}^{y}$, are degenerate (shown in Fig. 2). The normalized propagation constant versus normalized wave vectors is shown in Fig. 2. We compute only the first three eigenvalues at each given frequency. The average CPU time is about $14 \mathrm{~s}$. As the 


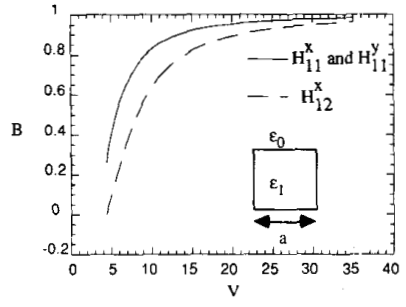

Fig. 2. A square waveguide. Normalized propagation constant $B=\left((\beta / k)^{2}\right.$ $\left.-\epsilon_{0}\right) /\left(\epsilon_{1}-\epsilon_{0}\right)$ versus normalized wave vector $V=k a\left(\epsilon_{1}-\epsilon_{0}\right)^{1 / 2}, k$ is free space wave vector, $a=1 \mu \mathrm{m}, \epsilon_{1}=13.1 \epsilon_{0}$.

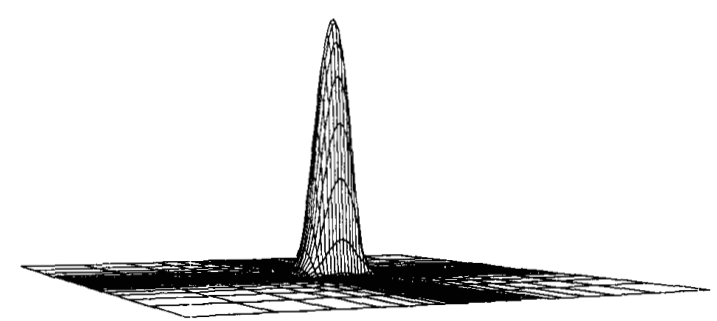

Fig. 3. The distribution of $H_{x}$ for the first mode for the square dielectric waveguide.

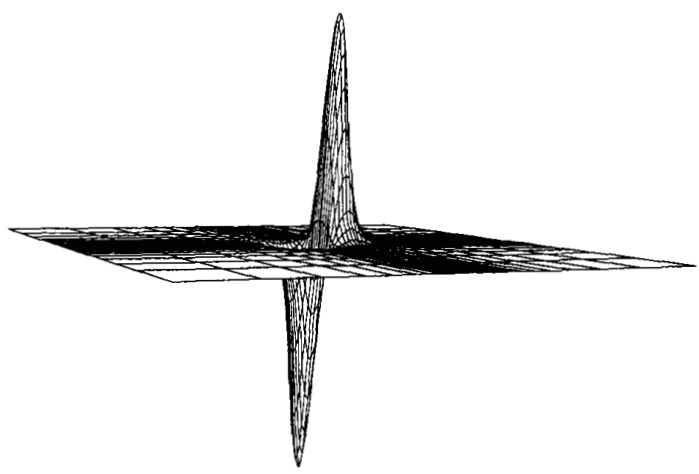

Fig. 4. The distribution of $H_{x}$ for the second mode for the square dielectric waveguide.

frequency decreases, the waveguide tends to cut off all the guided modes. Our calculation for this structure without the use of the inverse power method converges rapidly, and yields a norm of residual less than $10^{-11}$, where the norm of residual is defined as $\|A x-\lambda x\|$. It agrees with the prediction from the modified Arnoldi method [9], [14]. Our results also agree wih the previously published results from the different numerical approaches [5]-[7]. Figs. 3 and 4 show the $H_{x}$ component distribution for the first mode and $H_{x}$ component for the second mode. They are well confined in the guided region, and rapidly decaying in the clad layers.

The second example is a channel waveguide, Fig. 5 shows the waveguide structure and the normalized propagation constant versus the normalized wave vectors. The first three modes are computed in microwave length range. As the frequency decreases, the first two propagation constants separate even

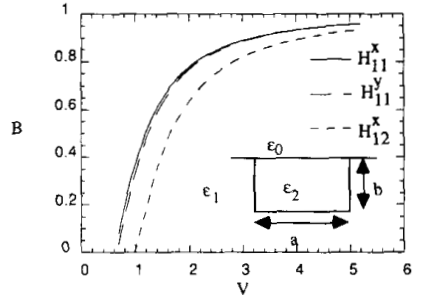

Fig. 5. A channel waveguide. Normalized propagation constant $B=\left((\beta / k)^{2}-\epsilon_{1}\right) /\left(\epsilon_{2}-\epsilon_{0}\right)$ versus normalized wave vector $V=k a\left(\epsilon_{2}-\epsilon_{0}\right)^{1 / 2} \cdot k$ is free space wave vector, $a=2 b=6 \mu \mathrm{m}$, $\epsilon_{1}=2.13 \epsilon_{0}, \epsilon_{2}=2.25 \epsilon_{0}$.

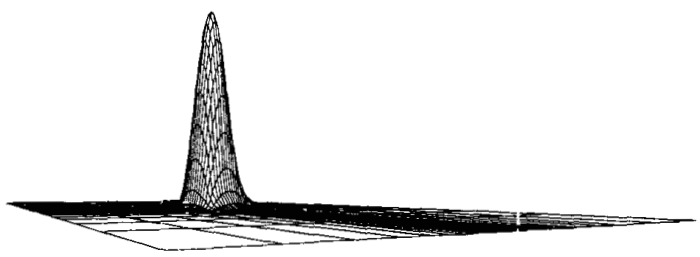

Fig. 6. The distribution of $H_{x}$ for the first mode for the channel waveguide.

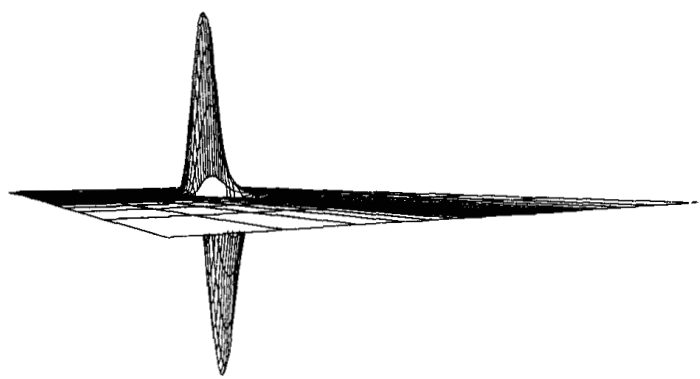

Fig. 7. The distribution of $H_{x}$ for the second mode for the channel waveguide.

more, and $H_{11}^{y}$ mode is cut off first. As the frequency increases, the first two modes of the $H_{x}$ are getting close to each other. Figs. 6 and 7 show the $H_{x}$ component distribution for the first two modes. The average CPU time is about $17 \mathrm{~s}$ for one set of eigenvalues at each given frequency. Our results agree well with previously reported results [5], [7].

The last example is a single mode quantum well ridge waveguide (see Fig. 8). This structure has weak guided modes and the guided region is much smaller than the clad layers. The numerical results are shown in Fig. 9, the normalized propagation constant versus normalized the wave vectors. Fig. 10 shows the $H_{x}$ component distribution of the first mode. The field is weakly confined in the lateral direction. Our calculation for this structure needs the acceleration by the inverse power method [11] to improve the convergence. The maximum norm of residual is less than $10^{-8}$ for the first two modes. The average CPU time is about $1 \mathrm{~min}$.

\section{CONCLuSIONS}

The technique we presented in this paper is including the physical reality of hybrid modes as well as the coupling between them, and sufficiently flexible for complex structures 


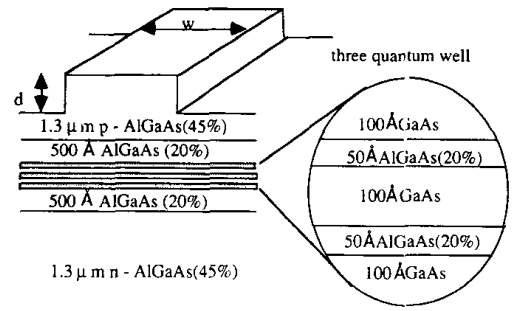

Fig. 8. A quantum well single-mode ridge waveguide, $t=3 \mu \mathrm{m}$, $d=0.9 \mu \mathrm{m}$.

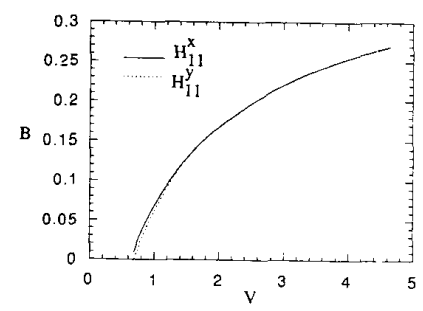

Fig. 9. Normalized propagation constant $B=\left((\beta / k)^{2}-\epsilon_{\text {clad }}\right) /\left(\epsilon_{\text {core }}\right.$ $\left.-\epsilon_{\mathrm{clad}}\right)$ versus normalized wave vector $V=d k\left(\epsilon_{\mathrm{core}}-\epsilon_{\mathrm{clad}}\right)^{1 / 2} . k$ is free space wave vector, $\epsilon_{\mathrm{clad}}$ is the maximum dielectric constant in the clad layers, the $\epsilon_{\mathrm{cor}}$ is the maximum dielectric constant in the guided region, and $d$ is the thickness of the core.

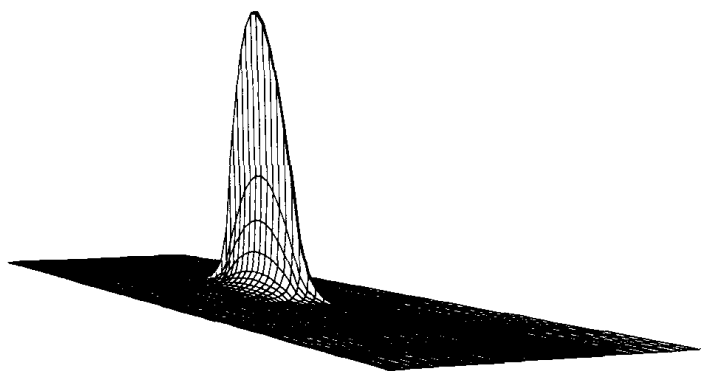

Fig. 10. The distribution of $H_{x}$ for the first mode for the quantum well single-mode waveguide.

of dielectric waveguides. We have proved the efficiency, reliability, and accuracy for this technique by testing suitable examples.

The first two examples show the multiple-mode structure, and the modes are strongly guided. The propagation constants are close to the product of maximum dielectric constant and wave vector at most frequency region but the cut-off region. The corresponding matrices tend to be diagonal dominant, and the magnitude of the negative eigenvalues is two orders bigger than the positive eigenvalues. The convergence of computations for the eigenvalues is fast, without using an inverse power method. The last example shows a typical structure of a quantum well single mode waveguide structure, where the guided region is narrow. The optical field confinement of the structure is small, about $7 \%$. The single mode is weakly guided in this structure, and the rapid decaying modes are very dominant. The magnitude of the negative eigenvalues is about six orders bigger than the positive eigenvalue. A relatively large number of nodes is required for the convergence of eigenvectors. For such type of waveguide structure, efficient, reliable, and accurate numerical technique is demanded.

The numerical technique is suitable for a very large and sparse matrix. It is also very flexible with discretization techniques. For a different discretization technique, we only need to change a subroutine for the matrix multiplication. This numerical technique does not use any preconditioning. It is presumed that this method will be more powerful with preconditioning either in the Arnoldi method (Polynomial) or in the inverse iteration (ILU). Currently we use this method to analyze step-index profile dielectric waveguides. It is also suitable for continuously variable-index profile waveguides, by using the first-order approximation of a Taylor expansion of the index.

\section{REFERENCES}

[1] E. Goell, "A circular-harmonic computer analysis of rectangular dielectric waveguides," Bell Syst. Tech. J., vol. 48, pp. 2133-2160, 1969.

[2] B. M. A. Rahman and J. B. Davies, "Penalty function improvement of waveguide solution by finite elements," IEEE Trans. Microwave Theory Tech., vol. MTT-32, pp. 922-928, 1984.

[3] K. Hayata, "Vectorial finite-element method without any spurious solutions for dielectric waveguiding problems using transverse magneticfield component," IEEE Trans. Microwave Theory Tech., vol. MTT-34, pp. 1120-1124, 1986.

[4] Z. Abid, K. Johnson, and A. Gopinath, "Analysis of dielectric guides by transverse magnetic field finite element," to appear in J. Lightwave Technol.

[5] K. Bierwirth, N. Schulz, and F. Arndt, "Finite-difference analysis of rectangular dielectric waveguide structure," IEEE Trans. Microwave Theory Tech., vol. MTT-34, pp. 1104-1114, 1986.

[6] N. Schulz, K. Bierwirth, and F. Arndt, "Finite-difference analysis of integrated optical waveguide without spurious solutions," Electron. Lett., vol. 22 , pp. 963-965, 1986.

[7] A. Galick, T. Kerhoven, and U. Ravaioli, "Iterative solution of the eigenvalue problem for a dielectric waveguide," IEEE Trans. Microwave Theory Tech., vol. MTT-40, pp. 699-705, 1992.

[8] R. Varga, Matrix Iterative Analysis. Englewood Cliffs, NJ: PrenticeHall, 1962.

[9] W. E. Arnoldi, "The principle of minimized iterations in the solution of the matrix eigenvalue problem," Quart. Appl. Math., vol. 9, pp. 17-29, 1951.

[10] Y. Saad, Partial Eigensolutions of Large Nonsymmetric matrices Research Report, YALEU/DCS/RR-397, 1985.

[11] G. Golub and C. van Loan, Matrix Computations. Baltimore: John Hopkins University Press, 1989.

[12] A. T. Chronopoulos, "S-step iterative methods for (non)symmetric (in)definite linear systems," SIAM J. Num. Anal., vol. 28 , no. 6 , pp. $1776-1789,1991$.

[13] J. S. Hornsby and A. Gopinath, "Numerical analysis of a dielectricloaded waveguide with a microstrip line-finite difference methods," IEEE Trans. Microwave Theory Tech., vol. MTT-17, pp. 684-690, 1960.

[14] K. Kim and A. T. Chronopoulos, "An efficient parallel algorithm for extreme eigenvalues of sparse nonsymmetric matrices," The Int. J. on Supercomputing, vol. 6, no. 1, pp. 98-111, 1992.

[15] D. Yevick and B. Hermansson, "Split-step finite difference analysis of rib waveguides," Electron. Letters, vol. 25, no. 7, pp. 461-42, 1989.

[16] W. Huang, C. Xu, S. T. Chu, and S. K. Chaudhuri, J. Lightwave Technol. vol. 10, no. 3, pp. 295-305, 1992.

[17] Y. Chung and N. Dagli, "Analysis of $Z$-invariant and $Z$-variant semiconductor rib waveguides by explicit finite difference beam propagation method and nonuniform mesh configuration," IEEE J. Quantum Electron., vol. 27 , no. 10 , pp. 2296-2305, 1991. 
Haozhe Dong received the M.S. degree in chemical physics in 1990 and currently is working on the Ph.D. degree in Department of Electrical Engineering at the University of Minnesota. His current interests are in design, modeling, fabrication, and characterization of high-speed optoelectronic devices.

$\mathrm{Mr}$. Dong is a member of the OSA.

Anthony Theodore Chronopoulos received the Ph.D. degree in computer science at the University of Illinois in Urbana-Champaign in 1987.

$\mathrm{He}$ is an Assistant Professor in the Computer Science Department at the University of Minnesota. He has published over 20 technical papers in the areas of scientific computing and parallel processing. His current work focusses on parallel algorithms and its applications to solve engineering problems.
Junping Zou received the B.S. degree in semiconductor physics in 1982 , the M.S. degree in solid-state physics in 1985 from Sichuan University and the Ph.D. degree in electrical engineering in 1991 from the University of Minnesota, where she conducted the design, fabrication, modeling, and characterization of heterostructure field effect transistors.

Since 1991 she has been with Cray Research Inc. Her current interest are in MCM design and fabrication, and electronic and optoelectronic device modeling.

Anand Gopinath, photograph and biography not available at the time of publication. 